Please do not remove this page

RMIT

UNIVERSITY

\title{
Crystallization kinetics of polydisperse colloidal hard spheres: experimental evidence for local fractionation
}

Martin, Stephen; Bryant, Gary; Van Megen, William

https://researchrepository.rmit.edu.au/esploro/outputs/9921859256201341/filesAndLinks?institution=61RMIT_INST\&index=null

Martin, S., Bryant, G., \& Van Megen, W. (2003). Crystallization kinetics of polydisperse colloidal hard spheres: experimental evidence for local fractionation. Physical Review E, 67(6),

061405-1-061405-061411. https://doi.org/10.1103/PhysRevE.67.061405

Document Version: Accepted Manuscript

Published Version: https://doi.org/10.1103/PhysRevE.67.061405

Repository homepage: https://researchrepository.rmit.edu.au

(C)2003 The American Physical Society

Downloaded On 2023/04/26 22:34:46 +1000 
Thank you for downloading this document from the RMIT Research Repository.

The RMIT Research Repository is an open access database showcasing the research outputs of RMIT University researchers.

RMIT Research Repository: http://researchbank.rmit.edu.au/

\author{
Citation: \\ Martin, S, Bryant, G and Van Megen, W 2003, 'Crystallization kinetics of \\ polydisperse colloidal hard spheres: experimental evidence for local fractionation', \\ Physical Review E, vol. 67, no. 6, pp. 061405-1-061405-11.
}

See this record in the RMIT Research Repository at:

https://researchbank.rmit.edu.au/view/rmit:3794

Version: Accepted Manuscript

\title{
Copyright Statement:
}

(C) 2003 The American Physical Society

Link to Published Version:

http://dx.doi.org/10.1103/PhysRevE.67.061405 
Please cite as:

Martin, S., Bryant, G., Van Megen, W.

"Crystallization kinetics of polydisperse colloidal hard spheres: Experimental evidence for local fractionation," Physical Review E - Statistical, Nonlinear, and Soft Matter Physics 67, 061405/1-061405/11 (2003). 


\title{
Crystallization kinetics of polydisperse colloidal hard spheres: Experimental evidence for local fractionation
}

\author{
S. Martin, ${ }^{1, *}$ G. Bryant,${ }^{1, \dagger}$ and W. van Megen ${ }^{1, \ddagger}$ \\ ${ }^{1}$ Department of Applied Physics, Royal Melbourne Institute of Technology, \\ GPO BOX 2476V Melbourne, 3001 Australia.
}

(Dated: June 10, 2003)

\begin{abstract}
We present the crystallization kinetics for two polydisperse hard sphere particle stocks with differing particle size distributions. One of the latexes had a relatively symmetrical distribution, the other had a more polydisperse distribution, which was highly skewed to smaller sizes. The emerging Bragg reflections from the crystallizing samples were measured using a technique that provides improved statistical averaging over our previous methods. It was observed that for the more polydisperse particles, the onset of nucleation was delayed by up to an order of magnitude in reduced time, and displayed qualitatively different growth behavior compared to the particles with the more symmetric size distribution. Based on these measurements and time lapse photographs we propose a growth mechanism whereby crystallisation occurs in conjunction with a local fractionation process near the crystal-fluid interface, which significantly alters the kinetics of crystallite nucleation and growth. This fractionation effect becomes more significant as polydispersity or skewness increases.
\end{abstract}

PACS numbers: 64.70.Dv, 81.10.Fq, 82.70.Dd

Keywords: crystallization, hard spheres, colloids, crystallization kinetics, polydispersity

*Electronic address: stephen.martin@rmit.edu.au

${ }^{\dagger}$ Electronic address: gary.bryant@rmit.edu.au

${ }_{\ddagger}^{\ddagger}$ Electronic address: bill.vanmegen@rmit.edu.au 


\section{INTRODUCTION}

Over half a century ago Kirkwood [1] speculated that systems with purely repulsive potentials would undergo a freezing transition. Subsequent computer simulations by Hoover and Ree [2] showed that a system of hard-spheres has a solid/fluid transition with the now accepted freezing and melting volume fractions of 0.494 and 0.545 respectively. More recently, it was observed by Pusey and van Megen [3] that colloidal particles used as model hard-sphere systems exhibit the predicted phase behaviour. Colloidal particles, manufactured with a diameter of about half a micrometre and a refractive index close to that of the suspending fluid, provide an ideal system for studying phase transitions, with visible light being the appropriate experimental probe. The size of the colloidal particles ensures that their motions are slow enough to study in real time, and makes practical measurements of the crystallisation kinetics, with colloidal crystals generally taking anywhere from a few minutes to several days to grow. The crystalline phase observed in colloidal hard-spheres is spectacularly opalescent, due to the presence of Bragg reflecting planes having spacings comparable to the wavelength of visible light. The planes have a stacking which is similar to a face-centred cubic (FCC) or hexagonal close-packed (HCP) structure with a high number of stacking faults [4].

There are two main spectroscopic methods that have been used to measure the kinetics of solidification. Small angle light scattering [5-7] which measures the density fluctuations in the crystallizing sample, and Bragg crystallography. Clark et al [8] first made time resolved Bragg measurements from a single body-centred cubic (BCC) crystal of charged colloidal particles. Subsequently Dhont et al. [9] measured time resolved Bragg scattering in a slightly charged colloidal system which exhibited many crystallites analogous to an atomic crystalline powder. This work introduced the basic methods which subsequent experiments followed [10-18]. These experiments all showed an initial period of rapid conversion of fluid to solid, followed by a decrease in conversion rate at later times.

This led to work by several authors to study crystallization kinetics, particularly on hard sphere systems. Despite the apparent simplicity of these systems, results from independent experiments on samples made from different particles do not always agree quantitatively (for example $[10,14,15]$ were studies performed under slightly different conditions and there were distinct differences among the results). It has been suggested that some differences are due 
to the effects of gravitational settling, and consequently time resolved measurements have been made under microgravity conditions $[12,15,16,19]$. These measurements, as well as other results [20], show that gravitational forces on the crystals do indeed influence the crystal structure. Comparison of kinetic data for a sample with and without the effect of gravity [16] shows that gravitational forces influence the growth in the number and size of individual crystallites in a crystallizing sample. However, the total rate of conversion from fluid to solid within the sample shows no change in growth rates or characteristic crossover times when the influence of gravity is reduced (see figure 3 of ref [16]). Thus gravity alone is not sufficient to explain the significant quantitative differences between the data in references $10,14,15$ and the results presented in this paper.

There is straightforward evidence from computer simulations and experiment that polydispersity has a significant influence on crystallisation kinetics [21]. Monte Carlo simulations from Kofke and Bolhuis [22] calculated a coexistence phase-diagram for crystallization in polydisperse systems, which showed fractionation as a possibility. In the phase diagram produced, the polydispersity in the crystal never exceeds a value of $\approx 6 \%$, indicating the presence of an upper limit to the polydispersity tolerance in a single hard-sphere crystal. Experimentally, colloidal samples of binary mixtures (radius ratio 0.85) measured by Henderson [23], suggest a preference for larger than average size particles in the forming crystals. These experiments also show that the inclusion of only a slight amount of a second component slowed down the solidification process [13]. Recent simulations and theory addressing this issue include work by Bartlett [24], who has suggested that the fractionated crystal is the equilibrium annealed state in a polydisperse hard sphere system, Evans and Holmes [25], who predict that the the mean size of colloidal particles incorporated into a crystal is smaller than that predicted by equilibrium calculations, and Auer and Frenkel [26], who have demonstrated that the presence of polydispersity leads to a maximum in the probability of formation of critical nuclei as the supersaturation is increased. There is a need for more experimental work to address the issues raised by these and related works.

In this paper we present qualitative observations from time lapse photographs and detailed static structure factor measurements from two different latexes with differing particle size distributions. We then present data from time resolved Bragg scattering measurements on each latex. We describe a mechanism by which local fractionation of the fluid can account for the slowing down of the solidification process. We explain our observations by providing 
a model by which solidification proceeds in polydisperse colloidal samples via a fractionation process [27]. Some of the structural data has been presented elsewhere [28].

\section{METHOD}

The particles used in this study consist of a copolymer core of methylmethacrylate (MMA) and tri-fluoroethylacrylate (TFEA). Different concentrations of TFEA give different refractive indices of the particle core. The amount of TFEA in the particles can then be adjusted, during manufacture, to allow the particle cores to have the same refractive index as the suspension solvent, cis-decalin $\left(\mathrm{n}=1.483 @ 25^{\circ} \mathrm{C}\right)$, at a practical range of temperatures [29]. The two latexes described here (labelled $\mathrm{X}$ and $\mathrm{W}$ ) were made so that their refractive indices match the solvent at temperatures of $9^{\circ} \mathrm{C}$ and $24^{\circ} \mathrm{C}$ respectively (see table I). To prevent coagulation of the particles, a stabilising barrier of poly-12-hydroxystearic acid (PHSA), 10 nm thick, was chemically bonded to the surface. Samples will be identified by their latex type, followed by the sample volume fraction in brackets (i.e. X[0.55] denotes a sample of latex $\mathrm{X}$ at a volume fraction of $\phi=0.55)$.

The mean particle core radius and polydispersity were determined by analysis of measurements of the scattered intensity and apparent radii as functions of angle as described by Bryant et al. [31]. The size distributions for the two latexes are shown in figure 1. Figure 1(a) shows latex $\mathrm{X}$ to have a slightly negatively skewed distribution, which was fit using a Weibull distribution [31], with polydispersity, $\sigma \approx 6.8 \%$. For latex $\mathrm{W}$, a single skewed distribution was insufficient, so a fit was made from a combination of two Gaussian functions (figure 1(b)). One with $R_{a v}=245 \mathrm{~nm}, \sigma=4$, and another very broad Gaussian encompassing particle sizes $<250 \mathrm{~nm}$. It should be noted that this highly skewed (or even pseudo bimodal) distribution found for latex $\mathrm{W}$ is not a unique fit [31], but the results of both light scattering and sedimentation studies suggest this to be the most likely distribution.

These particles have phase behaviour compatible with that of a simple hard sphere system [3]. The hardness of the interaction has recently been confirmed by direct measurements [32]. From sedimentation experiments the apparent melting and freezing volume fractions are identified, and then scaled to an effective hard sphere volume fraction by scaling the measured freezing volume fraction to the theoretical value of 0.495 . The effective melting volume fraction $\phi_{\text {melting }}$ can then be determined $[3,33]$ (see table I). 
The spectrometer (Figure 2) consists of a sample situated in a hemispherical glass vat, which contains cis-decalin to provide index matching with the sample. The sample is illuminated with collimated laser light $(\lambda=532 \mathrm{~nm})$ which is shaped through an aperture then aligned along the optical axis of the system by two adjustable mirrors. The hemispherical vat, containing the sample, acts as a lens which focuses the scattered light onto two diode array cameras placed on opposite sides of the hemisphere, at the focal point, and set at an angle, $\theta$. In this work the angle was chosen so that the detector window captures the interlayer (Bragg) reflection between close-packed planes. This reflection occurs at $q R \approx 3.6$, where $R$ is the average particle radius and $q$ is the scattering vector given by:

$$
q=\frac{4 \pi n}{\lambda} \sin \left(\frac{\theta}{2}\right)
$$

where $n$ is the refractive index of the solvent. The detectors are mounted on an arm which can be rotated about the optical axis of the system (shown by the angle $\varphi$ in figure 2 ). At $q R \approx 3.6$, for the particles used here, each detector spans a $q$ range of $\sim 3 \mu \mathrm{m}^{-1}$. Integration over the full Debye-Sherrer ring captures about 400 times as many crystal orientations as the (more conventional) planar spectrometer. Of course this additional averaging over orientations could be achieved more easily by rotating the sample about the vertical axis. However, due to the extreme fragility of colloidal crystals, it is not desirable to rotate the sample in order to improve the orientational average. This method also allows for the observation of any systematic variations that might exist around the Debye-Scherrer ring. The apparatus has a facility for tumbling of the sample in situ, minimizing temperature gradients and allowing data collection at the earliest possible times. A detailed description of the apparatus has been published elsewhere $[17,18]$. A limitation of the apparatus is that the detectors can only be positioned to an accuracy of $\sim 1^{\circ}$. To reduce the absolute uncertainty in $\mathrm{q}$ introduced by this positional error, the maximum of the peak in a fully crystallized sample was measured, and this value was compared to the angle measured from the same sample in the planar goniometer (previously used by Harland [10] and Henderson [23]) which has a more accurate angle measurement scale. Calibration of the data this way gives accuracy in angle of $\sim \pm 0.1^{\circ}$ (corresponding to $q R$ uncertainty of \pm 0.01 within the range of the detector window centred on the main Bragg reflection).

Time is expressed either in real time, or in units of the Brownian time, $\tau_{b}=R^{2} / D_{o}$, where $R$ is the average particle radius of the latex being measured and $D_{o}$ is the free particle 
diffusion coefficient. The Brownian times are given in table I. Prior to measurement, each sample was tumbled at a frequency of $\approx 1 \mathrm{~Hz}$ to shear melt the sample. After a few minutes of this action the sample appeared amorphous and showed no evidence of Bragg reflections. In order to ensure no residual crystals were present, all samples were tumbled much longer than this (several hours) prior to each measurement. After tumbling was stopped, and the sample was locked into a vertical position, 30s were allowed for the shear flows within the sample to dissipate. After this wait, we define time $\tau_{o}=0$, as the start of the experimental run. Measurements of the scattered intensity were take at regular intervals up to a time $\tau_{f}$ (corresponding to $\approx 60$ hours) for each sample. Beyond $\tau_{f}$ samples showed little change in the scattered intensity. Moreover, up to $\tau_{f}$ there was no evidence of sedimentation (see section III for a discussion of gravitational effects).

The method used to isolate the Bragg reflection from the scattered intensity is the same as that outlined by Harland [10], and subsequently adopted by a number of other authors $[14,15]$. The Bragg reflection being studied is that due the stacking of hexagonal planes (in crystallographic notation the $\mathrm{FCC}(111)$ or $\mathrm{HCP}(002)$ reflection). The structure factor $S(\varphi, q, \tau)$, as a function of angle around the optical axis, $\varphi$, scattering vector, $q$ and time, $\tau$, can be expressed as

$$
S(\varphi, q, \tau)=\frac{I(\varphi, q, \tau)}{I(\varphi, q, 0)} \times S_{p-y}(q, \tau)
$$

where $I$ is the scattered intensity and $S_{p-y}(q, \tau)$ is the Percus-Yevick structure factor. From equation 2, the contribution to the structure factor from the Bragg reflecting crystallite planes, $S_{c}(\varphi, q, \tau)$, is isolated using

$$
S_{c}(\varphi, q, \tau)=S(\varphi, q, \tau)-\beta S(\varphi, q, 0)
$$

Where $\beta$ is a scaling factor. The final structure factor peaks are fitted with Gaussian functions, from which the area under the peak, peak width and peak position are determined. For more details about the analysis see [10, 17].

Results presented are based on the average of $S(\varphi, q, \tau)$ around the Debye-Scherrer cone, i.e. by integrating over $\varphi$. The area under the main reflection, $X(\tau)$, hereafter referred to as the crystallinity, is proportional the amount of sample in the scattering volume which has been converted to a Bragg reflecting solid phase;

$$
X(\tau)=\int S_{c}(q, \tau) d q
$$


No attempt was made to normalise $X(\tau)$, which was calculated from the width, $\sigma(\tau)$ of the Gaussian fit via;

$$
X(\tau)=\sqrt{\frac{2 \pi}{\sigma(\tau)^{2}}}
$$

The average linear dimension of the crystals, $L(\tau)$, is given by:

$$
L(\tau)=\frac{\pi K}{\sigma \sqrt{2 \ln 2}}
$$

where $K=1.0747$ is the Scherrer constant for a spherical shaped crystal [35]. When the Bragg reflection is from the close packed planes, then the position of the peak maximum $q_{\max }(\tau)$ gives the volume fraction of the crystal phase $\phi_{c}(\tau)$

$$
\phi_{c}(\tau)=\frac{\left.2\left(q_{\max }(\tau) R\right)^{3}\right)}{9 \sqrt{3} \pi^{2}}
$$

The scattering volume, $\mathrm{V}$ (typically $600 \mathrm{~mm}^{3}$ but varying for each run), and $X(\tau)$ along with the average linear dimension of the crystals can be used to calculate an estimate of the number of crystallites in the scattering volume, $n(\tau)$.

$$
n(\tau)=\frac{X(\tau) V}{L(\tau)^{3}}
$$

The number of crystallites in the sample is expressed in reduced units of number density, $N(t)$, given by

$$
N(\tau)=\frac{n(\tau) R^{3}}{V}
$$

\section{RESULTS}

\section{A. Effects of Gravity}

Samples will be referred to by their series label (table I) followed by their volume fraction in brackets. We will begin by addressing the gravitational issues. The particle Peclét value is given by $P_{e}=\tau_{s} / \tau_{b}$, where $\tau_{s}$ is the time for a free particle to sediment one radius, $\tau_{s}=U_{o} / R$, where $U_{o}$ is the dilute sedimentation velocity given by

$$
U_{o}=\frac{2 \Delta \rho R^{2} g}{9 \eta}
$$

where $\Delta \rho$ is the difference in density between the particle and solvent, $g$ is acceleration due to gravity and $\eta$ is the solvent viscosity. For latexes $\mathrm{X}$ and $\mathrm{W}$ the particle Peclét values are 
0.04 and 0.01 respectively. For a volume fraction of $\approx 0.5$ we scale the sedimentation velocity $U / U_{o}$ by 0.05 (from figure 8 of Ackerson et al. [34]) and scale the long time diffusion, $D / D_{o}$ by 0.01 (from figure 8 of van Megen et al. [36]). The Peclét values at $\phi \approx 0.5, P_{e}(0.5)$, are $\approx 6 \times 10^{-5}$ for latex $\mathrm{X}$ and $\approx 10^{-4}$ for latex $\mathrm{W}$. In other words the effects of gravity are insignificant compared to the dynamics of the particles, so we can ignore the presence of gravity during the crystallization process. However, as mentioned previously, gravity has been shown to have a significant effect on the structure of grown crystals so we also need to quantify the effects of the stress from viscous flows, through crystal sedimentation.

In consideration of this, we have used the same equations as Zhu et al. [12] for calculating the critical radius, $R_{\text {crit }}$, at which viscous stresses applied by the fluid on a sinking crystal start to exceed the yield stress of the crystal and cause it to break apart. We have used the result from Zhu et al. and re-scaled it for our values of particle radius, solvent density and $\Delta \phi=\phi_{\text {freezing }}-\phi_{\text {melting }}$ (from table I) to obtain estimates of critical radii for our suspensions. The results give a critical radius of $\approx 9.5 h$ for latex $\mathrm{X}$ and $\approx 20 h$ for latex $\mathrm{W}$ ( $h$, the characteristic gravitational length of the particles, being $\approx 9 \mu m$ for latex $\mathrm{X}$ and $\approx 19 \mu \mathrm{m}$ for latex $\mathrm{W})$. This leads to an approximate size limit due to gravity of $85 \mu \mathrm{m}$ for latex X and $380 \mu \mathrm{m}$ for latex W. Until observed crystallites start to approach these sizes in our samples, we can neglect the effects of crystal sedimentation from our analysis of the growing crystallites.

\section{B. Direct Observations}

There are upper and lower limits on the volume fraction beyond which crystallization studies of the present samples are no longer practical. The lowest volume fraction studied for both the samples was $\phi=0.52$. For $\mathrm{W}[0.52]$ crystallites were visible, but there were too few crystals to obtain reliable averaging of the time dependent parameters. This places a lower limit on $\phi$ for this study. Neither latex showed growth of crystallites at $\phi>0.56$ over the experimental timeframe. However subtle shifts in the structure factor peak at late times showed evidence of restructuring taking place in the fluid in latex X. Note that the latex used by Harland [10] (which had 5\% polydispersity) showed homogeneous nucleation up to $\phi_{g} \approx 0.575$. But for $\phi>\phi_{g}$ larger (heterogeneous) crystals formed (as shown in [37]). These observations, combined with those of Henderson et al. [38], show that crystallization 
of colloids at $\phi$ near $\phi_{g}$ is extremely sensitive to polydispersity.

Samples of latex X and W particles show qualitatively different modes of crystallization, and time lapse photographs can be viewed online [39]. We give a brief description here. In latex $\mathrm{X}$ at early times a large number of small crystallites appear uniformly distributed throughout the sample. As time progresses, these same crystals grow in size at approximately the same rate to fill the entire sample volume. In latex $\mathrm{W}$ at early times fewer small crystal regions are seen, scattered randomly throughout the sample volume. These initial crystallites continue to grow rapidly to some maximum size (within 2-3 hours). After this initial burst of crystallite growth, more small crystal regions are seen to form within the sample, which then also continue to grow. Other volume fractions show similar behaviour.

\section{Final Structure Factors}

Figure 3 shows the structure factors in the detector window at time $\tau_{0}$ (sample still fluid) and $\tau_{f}$ (at the end of the experiment). In latex $\mathrm{X}$ three distinct peaks are observed and the main reflection (from the close packed planes) appears at, or to the left of, the fluid peak. The inter-layer spacing in the crystallites is thus equal to or greater than the average spacing of particles in the metastable fluid. The results of latex $\mathrm{X}$ can be interpreted in terms of random stacking of hexagonal planes $[4,28]$, i.e. a mixture of $\mathrm{HCP}$ and FCC. By contrast, latex $\mathrm{W}$ shows only a single peak within the detector window, positioned at greater $q$ than the fluid peak. The emergence of only one very strong reflection in the $\mathrm{W}$ samples indicates that the solid phase is ordered in one direction only.

Both latexes were also studied on a planar crystallization spectrometer with greater angular range, and the results are shown in figure 4. Changing the particle/solvent contrast (by means of temperature control) alters the particle form factor. This was used to highlight or hide intensity peaks by changing the position of the minimum in the particle form factor. At no contrast were other reflections visible near the primary peak in the $\mathrm{W}$ samples. At higher angles $(q R>5)$, some small reflections were seen, but were randomly scattered and could not be indexed to any specific crystal structure. Note that this spectrometer integrates over only $\sim 1^{\circ}$ in $\varphi$, so the data is necessarily much less reliable than the data in figure 3 .

The main peak for latex $\mathrm{W}$ lies between the peak from close-packed planes (FCC(111)) and the $\mathrm{HCP}(101)$ peak. As a pure HCP structure is never observed in colloidal systems, 
and there are no other ordered reflections, it is concluded that the main peak is from a stacking of close-packed planes, but is at higher $q R$ than the expected value due to the lack of registration of the planes (for more detail see Martin et al. [28]).

Figure 5 shows the final crystal structure peaks for both latexes at 2 different volume fractions for the 32 angular segments around the Debye-Scherrer cone. Figure 6 shows $X\left(\varphi, \tau_{f}\right), \sigma\left(\varphi, \tau_{f}\right)$ and $q_{\max }\left(\varphi, \tau_{f}\right)$ for the same samples plotted as a function of the position of the detector, $\varphi$. From both graphs it can be seen that for latex $\mathrm{W}$ there is considerable variation with angle $\varphi$. From studying similar data for repeat runs on the same sample, it was determined that the variation was random. This contrasts with systematic variations as a result of structural symmetry in the crystals that has been observed by Heymann et al. [14] . The large variation in the Bragg reflections of latex W (compared to the equivalent volume fraction for latex $\mathrm{X}$ ), combined with the observation of only one Bragg reflection, confirms the picture of latex $\mathrm{W}$ forming a solid phase consisting of unregistered planes. In a more ordered structure like latex X crystals, there are a higher number of close packed planes for a given volume of crystallite, leading to the reduced noise seen in Figure 5.

\section{Time Resolved Quantities}

Results for the crystallinity $X(\tau)$ are shown in figure 7. Qualitatively similar results have been obtained in other work by both Bragg (e.g. [11]) and small angle scattering(e.g. [6]). The period of rapid conversion has been attributed to nucleation and growth, and the late stage slow conversion to ripening. The crossover time $\tau_{c}^{X}$ between these two regimes is determined as shown in figure 8. Both the crossover time, and the time at which crystallites are first observed (the first data points for each series in figure 7), decrease with increasing volume fraction in latex $\mathrm{X}$. For latex $\mathrm{W}$, the crossover time gets smaller from $\mathrm{W}[0.53]$ to $\mathrm{W}[0.54]$, and increases again at the highest volume fraction, 0.55. In addition to these features, for X[0.55] and all three W samples, a period of a slower conversion precedes the rapid growth stage. This is highlighted in figure $7(\mathrm{~b})$, which shows data for latex $\mathrm{W}$ on an expanded scale.

Figure 9 shows the average crystallite size $L(\tau)$ as a function of time. As with the crystallinity data, the crossover times $\tau_{c}^{L}$ separating different growth regimes, are identified (superscript, $L$, denotes crossover time for the linear dimension data). The striking quali- 
tative difference between the two latexes is that $L(\tau)$ increases monotonically for latex $\mathrm{X}$, whereas for latex $\mathrm{W}, L(\tau)$ reaches a maximum, then starts to decrease. This decrease is more significant at lower volume fractions. From our earlier estimates of critical radius due to gravitational stresses, it is seen that the average crystallite sizes for all the samples are less than $R_{c}$ with the exception of $\mathrm{X}[0.52]$, which reaches $\approx R_{c}$. Thus, for this sample, the undulations seen in the data at long times may reflect the effects of gravitationally induced breakup of crystals.

Figure 10 shows the number of crystallites $N(\tau)$ (equation 9) in the sample, as a function of time. The trend seen with latex $\mathrm{X}$ is that the number of crystals starts to increase, then slowly declines after the sample sets into a 'ripening' stage. The growth rates relative to volume fraction appear to be consistent with what was visually observed in the samples. Latex $\mathrm{W}$ on the other hand shows the samples undergoing a rapid increase in the number of crystallites present.

Figure 11 shows $q_{\max }(\tau)$ for the samples as a function of time. The error bar on the final value gives the uncertainty in the absolute value of $q$ due to the angle calibration error (discussed in section II). However, the noise on the data points is insignificant. Latex X again shows behaviour consistent with that seen in previous studies [10], which indicate that crystallites expand with time. Crystallites in all latex $\mathrm{W}$ samples remain more compressed relative to latex $\mathrm{X} . \mathrm{W}[0.54]$ and $\mathrm{W}[0.55]$ also show an initial increase in $q R$ (i.e. the degree of compression decreases before increasing again).

\section{DISCUSSION}

The two latexes studied here show a range of different behaviours which need to be explained. In particular we seek an explanation for the following:

1. Why does latex $\mathrm{W}$ show strong crystallite formation but no registration of planes (figures 3,4$)$ ?

2. Why does latex $\mathrm{W}$ show evidence of multiple spawnings of crystallites?

3. Why is the onset of crystallization of latex $\mathrm{W}$ delayed relative to latex $\mathrm{X}$ (figure 7)?

4. How can the slow initial conversion rate seen for latex $\mathrm{W}$ (figure 7 ) be explained? 
We first look back on $L(\tau)$ (figure 9) and the time lapse photographs for a view of what is happening. From the photographs of latex $\mathrm{W}$ we observed multiple spawning of populations of crystallites at various stages throughout the experimental timeframe. This is consistent with what is observed with the maximum in $L(\tau)$ seen for latex $\mathrm{W}$ in figure 9 . The initial crystallites in the sample grow rapidly and contribute to the increase in the average crystal size. These crystals eventually stop growing and further crystals begin growing at later times (when $\log _{10} \tau$ is between 5.5 and 6), thus reducing the average crystal size. The behaviour described here is most significant in $\mathrm{W}[0.53]$.

For $\mathrm{X}[0.52]$ the maximum linear dimension is approximately equal to the calculated gravity limited critical radius. We attribute the slight drop in $L(\tau)$ at $\log _{10} \tau \approx 4.8$ to this effect. As the existing crystallites are unable to grow further, there must be new spawning of nuclei in order to satisfy the equilibrium balance between the solid/fluid phases, leading to the small drop in average size. As these new crystals grow, the average size returns to the critical value. For this reason we exclude $\mathrm{X}[0.52]$ in the following arguments and limit ourselves to the other samples which are not affected by gravitation constraints.

We propose an explanation for our observed results, based on considering the particle size distribution within the samples. We attribute the initial slow increase in crystallinity (seen in X[0.55] and all latex $\mathrm{W}$ samples) to slow crystal growth whose rate is limited by a fractionation process where the forming nuclei are "selecting" the particles to be included for further crystal growth. Qualitatively, one expects that the greater the polydispersity, the more rearrangement of different species is required to form a crystal with minimum strain. This is merely a generalization of the idea of demixing of species upon solidification of a binary mixture (see e.g. [24]). This expands on ideas introduced in our earlier experimental studies on binary mixtures with slightly different radii $[13,23,38]$, and is consistent with our previous simulation studies on such mixtures [40], which show that the path to equilibrium in these binary mixtures requires local fractionation of species.

In the following we discuss the differences in the crystallization of the two latex species in terms of fractionation or segregation of species. Figure 12(a) shows behaviour typical of latex $\mathrm{X} ; X(\tau)$ and $L(\tau)$ both increase monotonically with $\tau$ in a qualitatively similar manner. The ratio, $\tau_{c}^{L}: \tau_{c}^{X}$, of the cross over times from rapid to slow change, is close to unity (see inset). We infer that conversion to crystal is dominated by growth. 
This behaviour contrasts with that seen for latex W (Figure 12(b)). While $X(\tau)$ still increases monotonically with $\tau, L(\tau)$ exhibits a maximum. Moreover, the time of the maximum in $L(\tau)$ approximately coincides with a minimum in $N(\tau)$, at least for $\mathrm{W}[0.53]$ and $\mathrm{W}[0.54]$. Thus, the growth of the properties of the Bragg peak reflects what is observed directly; for $\tau<\tau_{c}^{X}$ crystallization (i.e. the increase in $X(\tau)$ ) is dominated by nucleation or spawning of crystallites.

Equation 8, used to estimate the number of crystals in the scattering volume, requires that we have the average volume of the crystals, or $\left\langle L^{3}\right\rangle$, approximating the crystallites to be cubic in shape. What we actually measure from the Bragg reflection is the average linear dimension, $\langle L\rangle$, so what is used in equation 8 is $\langle L\rangle^{3}$. If all the crystallites in the sample are approximately the same size (as with the latex X samples) the two different measures are approximately equal. If there is a distribution of crystal sizes (as with latex W samples $)<L^{3}>$ can be up to 2-3 times greater than $<L>^{3}$ [27]. For this reason it is not appropriate to assign too much significance to the details of the data in figure 10 . We note only that for $\mathrm{W}[0.53]$ and $\mathrm{W}[0.54]$ the number of crystallites increases by more than an order of magnitude, which is significantly more than the estimated uncertainty in the quantity.

The apparent volume fraction calculated from equation 7 is shown in figure $13 . \quad 13(\mathrm{a})$ shows the volume fraction calculated assuming a particle radius of $\mathrm{R}=320 \mathrm{~nm}$ for latex $\mathrm{X}$ (note that we do not apply equation 7 to latex W samples, as the crystallite structure is less clear, so the equation is probably invalid). This value was determined by letting $\mathrm{X}[0.55]$, which appeared to be the best behaved sample, sit undisturbed for a week. Then the angle of the main Bragg refection was measured and the effective hard sphere particle radius was set so the volume fraction equaled 0.55 , as would be ideally expected for this sample. Using this value of $R$, the apparent final volume fractions of the $\mathrm{X}[0.52-0.54]$ samples are of the order of 0.58 , well above the expected value of 0.545 in equilibrium.

In order to explain this we note from equation 7 , that $\phi_{c}$ is very sensitive to $R$. If we assume that the fractionation hypothesis holds, then we would expect the average radius of the particles incorporated into the crystal phase to change with time. At early times the value would be near the peak of the distribution, as particles with smaller (or larger) than average size are excluded. As the sample evolves, equilibrium would require that these particles must eventually be included in a crystal phase, and so the average radius of 
particles in the crystallites would change. To explore this, figure 13(b) shows the calculated volume fraction of the crystal if the average radius used in the calculations is allowed to vary with time. The volume fraction is fixed at an initial and final value, and is allowed to vary with time between these values, proportional to the cube of the average linear crystal size, $L(\tau)$ (figure 9). The starting value is fixed at $\phi=0.595$ (chosen as it is the approximate average value of the initial volume fractions of the crystal for all latex X samples calculated from $q_{\max }$ with a radius of $\left.320 \mathrm{~nm}\right)$. The final value was set at the expected crystal volume fraction from the ideal hard sphere phase diagram - 0.545 for samples in coexistence, and 0.55 for the sample at $\phi=0.55$. Although the experimentally measured $\phi_{m}$ (table I) differs from the theoretical value, the theoretical values have been used here for simplicity. The value chosen has little effect on the following analysis. For the fully crystalline sample, $\mathrm{X}[0.55]$, the radius used varied from $320 \mathrm{~nm}-319 \mathrm{~nm}$ (i.e. little change). For X[0.53 and $0.54]$ the average particle radius used varied from $320 \mathrm{~nm}-314 \mathrm{~nm}$, and for X[0.52] the radius varied from $324 \mathrm{~nm}-321 \mathrm{~nm}$. Note that the starting value is somewhat arbitrary, and what is important here is the change in $R$ needed to arrive at the correct final equilibrium volume fraction.

These values of average radius needed to correctly predict the final volume fraction support the fractionation idea. For X[0.55], most of the volume is expected to crystallize, therefore most of the particles will need to be used. The driving force to crystallization is high, but the particles are closely packed, so under these conditions there will be less fractionation during crystallization - most of the particles in the distribution will be forced into the crystal phase, and so the average radius of particles in the crystallites will change little with time. In the other latex $\mathrm{X}$ samples the average particle radius must decrease with time to obtain the correct final volume fraction. At these lower volume fractions the forming crystallites have more room to exclude the smaller particles at the early stages of crystallization, so the average radius of particles in the crystallites is near the peak of the distribution. At later times, in order to achieve equilibrium, the smaller particle must be incorporated into the crystallites, so the average radius decreases.

It must be noted that relatively small changes in the radius can produce large changes in the apparent volume fraction data and it would be possible to manipulate the data almost anyway one desires. Despite this caveat however, the results of this analysis are suggestive. Combined with the other data presented here, it strongly supports the idea 
that local fractionation is taking place within the crystallizing fluid as suggested from recent simulations [24]. However, these results do not agree with the predictions of Evans and Holmes [25] that the crystal will be composed of smaller than average sized particles. It appears from the results here that the crystallites begin their formation with average or larger than average sized particles, and smaller ones become incorporated into the crystal structure only later in the crystallization process.

Although it is inappropriate to conduct a similar analysis for latex W samples, the observations on latex $\mathrm{W}$ are consistent with the idea of fractionation of particles within the sample in order to form crystals. Latex $\mathrm{W}$ are the most heavily skewed polydisperse particles used, so it takes more time for a nascent solid phase to select particles of compatible size to start forming crystals, hence the longer waiting times before observable crystals form. The particles that are excluded from these initial crystals will be redispersed in the region between the crystals. It is possible that these excluded particles may then start forming crystals themselves, but their growth will likely be limited by the lower availability of particles. This would have the effect of reducing the measured average crystal size (as seen in figure 9). This effect is most noticeable in $\mathrm{W}[0.53]$, where there is less thermodynamic drive to crystallization, so the particles have more time to fractionate, producing a larger maximum size. The fractionation in turn filters the fluid to some extent and produces a further population of particles which contributes to a smaller average size at long times.

\section{CONCLUSION}

We believe that the limitation on the maximum crystal size (when gravitational affects can be considered insignificant), and the presence of multiple populations of crystals are due to fractionation of particles in polydisperse samples. This effect is enhanced where there is a significant amount of skewness towards smaller than average sized particles in the distribution. Although the phase diagrams for samples with higher polydispersity or skewness, or with lower volume fractions, require less total volume to crystallize, in order to form larger crystals the local volume around the individual crystals needs to be filtered to a suitable size distribution. The higher volume fraction samples grow smaller crystals so less filtering is required. The higher driving force for nucleation, and therefore the higher density of initial nucleation sites in these samples, presumably allows for particle selection 
on a smaller local scale, and a particle initially rejected from one nucleation site is likely to be not far away from a compatible nucleation site. This may also explain the tendency away from a coarsening process in the higher volume fraction samples; the crystals forming in the local vicinity have average particle sizes different enough to reduce the likelihood of combining with each other. Simplistic modelling of the apparent volume fraction data also suggests a strong tendency for crystals to form from particles at the larger end of the size distribution.

\section{ACKNOWLEDGEMENTS}

The authors would like to thank Phil Francis for technical support. This work was carried out with the help of the Australian Research Council, grant number A69701447. 
[1] J. G. Kirkwood, J. Chem. Phys. 7, 919 (1939).

[2] W. G. Hoover and F. H. Ree, J. Chem. Phys. 49, 3609 (1968).

[3] P. N. Pusey and W. van Megen, Nature (London) 320, 340 (1986).

[4] P. N. Pusey, W. van Megen, P. Bartlett, et al., Phys. Rev. Lett. 63, 2753 (1989).

[5] K. Schätzel and B. J. Ackerson, Phys. Rev. E 48, 3766 (1993).

[6] K. Schätzel and B. J. Ackerson, Phys. Rev. Lett. 68, 337 (1992).

[7] Y. He, B. J. Ackerson, W. van Megen, et al., Phys. Rev. E 54, 5286 (1996).

[8] N. A. Clark, A. J. Hurd, and B. J. Ackerson, Nature (London) 281, 57 (1979).

[9] J. K. G. Dhont, C. Smits, and H. N. W. Lekkerkerker, J. Colloid Interface Sci. 152, 386 (1992).

[10] J. L. Harland, S. I. Henderson, S. M. Underwood, et al., Phys. Rev. Lett. 75, 3572 (1995).

[11] J. L. Harland and W. van Megen, Phys. Rev. E 55, 3054 (1997).

[12] J. Zhu, M. Li, R. Rogers, et al., Nature (London) 387, 883 (1997).

[13] S. I. Henderson and W. van Megen, Phys. Rev. Lett. 80, 877 (1998).

[14] A. Heymann, A. Stipp, C. Sinn, et al., J. Colloid Interface Sci. 207, 119 (1998).

[15] Z. Cheng, P. M. Chaikin, J. Zhu, et al., Phys. Rev. Lett. 88, 015501 (2002).

[16] Z. Cheng, J. Zhu, W. B. Russel, W. V. Meyer and P. M. Chaikin, Appl. Optics 40, 4146 (2001).

[17] P. Francis, S. Martin, G. Bryant, et al., Rev. Sci. Instrum. 73, 3878 (2002).

[18] P. Francis, Master of Technology Thesis, Department of Applied Physics (Royal Melbourne Institute of Technology, Melbourne, 2001).

[19] W. B. Russel, P. M. Chaikin, J. Zhu, et al., Langmuir 13, 3871 (1997).

[20] W. K. Kegel and J. K. G. Dhont, J. Chem. Phys. 112, 3431 (2000).

[21] P. N. Pusey, in Liquids, "Freezing and the glass transition", edited by J.-D. Levesque (Les Houches, 1990).

[22] D. A. Kofke and P. G. Bolhuis, Phys. Rev. E 59, 618 (1999).

[23] S. Henderson, Ph.D. Thesis, Department of Applied Physics (Royal Melbourne Institute of Technology, Melbourne, 1999), p. 117.

[24] P. Bartlett, J. Phys.: Cond. Mat. 12, A275 (2000). 
[25] R. M. L. Evans and C. B. Holmes, Phys. Rev. E 64, 011404 (2001).

[26] S. Auer and D. Frenkel, Nature 413, 711 (2001).

[27] S. Martin, Ph.D. Thesis, Department of Applied Physics (Royal Melbourne Institute of Technology, Melbourne, 2002).

[28] S. Martin, G. Bryant, W, van Megen, submitted.

[29] S. M. Underwood and W. van Megen, Coll. Polym. Sci. 274, 1072 (1996).

[30] G. Bryant, T. Mortensen, S. Henderson, et al., J. Colloid Interface Sci. 216, 401 (1999).

[31] G. Bryant, S. Martin, B. Budi, et al., Langmuir 19, 616 (2003).

[32] G. Bryant, S. R. Williams, I. K. Snook, et al., Phys. Rev. E 66, 060501 (2002).

[33] S. E. Paulin, B. J. Ackerson, Phys. Rev. Lett. 64, 2663 (1999).

[34] B. J. Ackerson, S. E. Paulin, B. Johnson, et al., Phys. Rev. E 59, 6903 (1999).

[35] R. W. James, The Optical Principles of the Diffraction of X-rays. (Bell and Sons, London, 1967).

[36] W. van Megen, T. C. Mortensen, S. R. Williams, et al., Phys. Rev. E 58, 6073 (1998).

[37] W. van Megen, S. M. Underwood, Nature 362, 616 (1993).

[38] S. I. Henderson, T. C. Mortensen, S. M. Underwood et al., Physica A 233, 102 (1996).

[39] See EPAPS Document No. [number will be inserted by publisher ], for time lapse photographs showing the crystallization of $\mathrm{X}[0.54]$ and $\mathrm{W}[0.54]$. A direct link to this document may be found in the online article's HTML reference section. The document may also be reached via the EPAPS homepage (http://www.aip.org/pubservs/epaps.html) or from ftp.aip.org in the directory /epaps/. See the EPAPS homepage for more information.

[40] S. R. Williams, I. K. Snook, W. van Megen, Phys. Rev. E 64, 021506 (2001). 
TABLE I: Description of particles used in this study. In the text samples will be referred to by their series label (X or W) followed by the sample volume fraction in brackets.

\begin{tabular}{cccccc}
\hline \hline $\begin{array}{c}\text { Preparation reference } \\
\text { (series label) }\end{array}$ & Polymer Match & Temperature & $\begin{array}{c}\text { Mean core radius } \\
(\mathrm{nm})\end{array}$ & $\tau_{b}$ & $\phi_{\text {melting }}$ \\
\hline XL60 $(\mathrm{X})$ & MMA 16.9\%TFEA & $9^{\circ}$ & 300 & 0.586 & 0.552 \\
WVM7 $(\mathrm{W})$ & MMA 24.4\%TFEA & $24^{\circ}$ & 245 & 0.215 & 0.535 \\
\hline \hline
\end{tabular}




\section{Figure Captions}

FIG 1: Particle size distributions from fits to dynamic and static measurements of dilute suspensions of latexes (a) X and (b) W. At least two possible fits were able to be made to the latex $\mathrm{W}$ data, both of which consisted of a distribution around $245 \mathrm{~nm}$ and some component of much smaller sized particles. From other investigations of these particles [31] the continuous fit shown here is the most likely one to represent the true distribution within the sample.

FIG 2: Bragg scattering apparatus. Laser light is collimated, shaped and aligned before being incident on the sample contained in a hemispherical glass vat. The hemisphere focuses the scattered light to two CCD detectors, mounted at opposite ends of a rotating arm. The detectors are set at a desired scattering vector $q$, relative to the optical axis of the system (dot-dash line). The rotating arm is free to rotate around the optical axis (angle $\varphi$ ) in order to collect the full scattering from the Debye-Scherrer rings.

FIG 3: Structure factor peaks at times $\tau_{o}$ (thin line) and $\tau_{f}$ (bold line) for latex $\mathrm{X}$ at volume fraction (a) 0.53 , (b) 0.54 , (c) 0.55 and for latex $\mathrm{W}$ at volume fractions (d) 0.53, (e) 0.54 and (f) 0.55. At $\tau_{o}$ to the sample is still entirely in the fluid phase. At the end of the experiment, $\tau_{f}$, crystals have formed producing Bragg reflections within the experimental window.

FIG 4: Scattered intensity over accessible range for (thin line) X[0.54] and (bold line) $\mathrm{W}[0.54]$. All other volume fractions that underwent crystallisation were similar. The vertical lines show possible Bragg reflections, labelled with both HCP and FCC notation. As can be seen latex X crystallises with a random stacking of close planes. The main peak for latex W lies between the peak from close-packed planes (FCC(111)) and the $\mathrm{HCP}(101)$ peak.

FIG 5: Structure factors measured for samples (a)X[0.53], (b)W[0.53], (c)X[0.55] and (d)W[0.55] at 32 segments around the optical axis, $\varphi$, at time $\tau_{f}$.

FIG 6: The variation in the peak parameters (a) $X\left(\tau_{f}\right)$, (b) $\sigma\left(\tau_{f}\right)$ and (c) $q_{\max }\left(\tau_{f}\right)$ around the optical axis, $\varphi$, for; $\mathrm{X}[0.55](\times), \mathrm{X}[0.53]$ (squares), $\mathrm{W}[0.55]$ (diamonds) and $\mathrm{W}[0.53](+)$. Data sets are offset on the vertical axis for clarity.

FIG 7: Crystallinity, $X(\tau)$, as a function of log reduced time as measured from the area of the main Bragg reflection. The peak area in the $\mathrm{X}$ samples has been normalised to the final value of the peak area X[0.55]. The data for the latex $\mathrm{W}$ samples has been similarly normalised to the final value of the $\mathrm{W}[0.54]$ sample. Due to the normalisation, the absolute 
values of $X(\tau)$ for the $\mathrm{X}$ latexes cannot be compared to those of W latex. Figure 7(b) shows latex $\mathrm{W}$ data on an expanded scale.

FIG 8: Graphical representation of the measurement of the crossover time $\tau_{c}$ for the crystallinity data, $X(\tau)$. Linear fits are made to the early rapid growth stage and the later, slower growth stage. The crossover time is defined as the point where the two fits cross. Equivalent characteristic times can be determined from plots of the average linear dimension $L$ vs time.

FIG 9: Log average linear dimensions versus times. Errors are approximately the size of the data points.

FIG 10: Log relative number of crystals in samples. The number of crystals is calculated from the crystallinity data (equations 8-9), so normalization precludes a direct comparison between the absolute values of the two latexes (see caption figure 7).

FIG 11: Position of the maximum of the main Bragg reflection in units $q R$.

FIG 12: Crystallinity, $X(\tau)$, and average linear dimension, $L(\tau)$, data for (a) $\mathrm{X}[0.53]$ (open squares $X(\tau)$, filled squares $L(\tau)$ ) and (b) W[0.53] (crosses $X(\tau)$, squared crosses $L(\tau))$ over a decade in reduced time. The crossover times for the crystallinity and average size occur at approximately the same time for the $\mathrm{X}[0.53]$ sample, a trend seen in the other latex X samples. In $\mathrm{W}[0.53]$ the maximum in average crystal size occurs at the start of the rapid growth stage of the crystallinity. The inserted tables give the ratio of the crossover times in the average crystal size data to the crossover time in the crystallinity data. Only an approximate estimate can be made for $\mathrm{W}[0.53]$ as there is no linear region. The maximum is therefore taken as the crossover time.

FIG 13: (a) Volume fraction for the samples measured from the position of the main Bragg FCC(111) reflection. The final error bars show absolute error due to calibration of the detector positions. (b) The apparent volume fraction of the $\mathrm{X}$ samples, allowing the particle radius to vary with time (see text). 

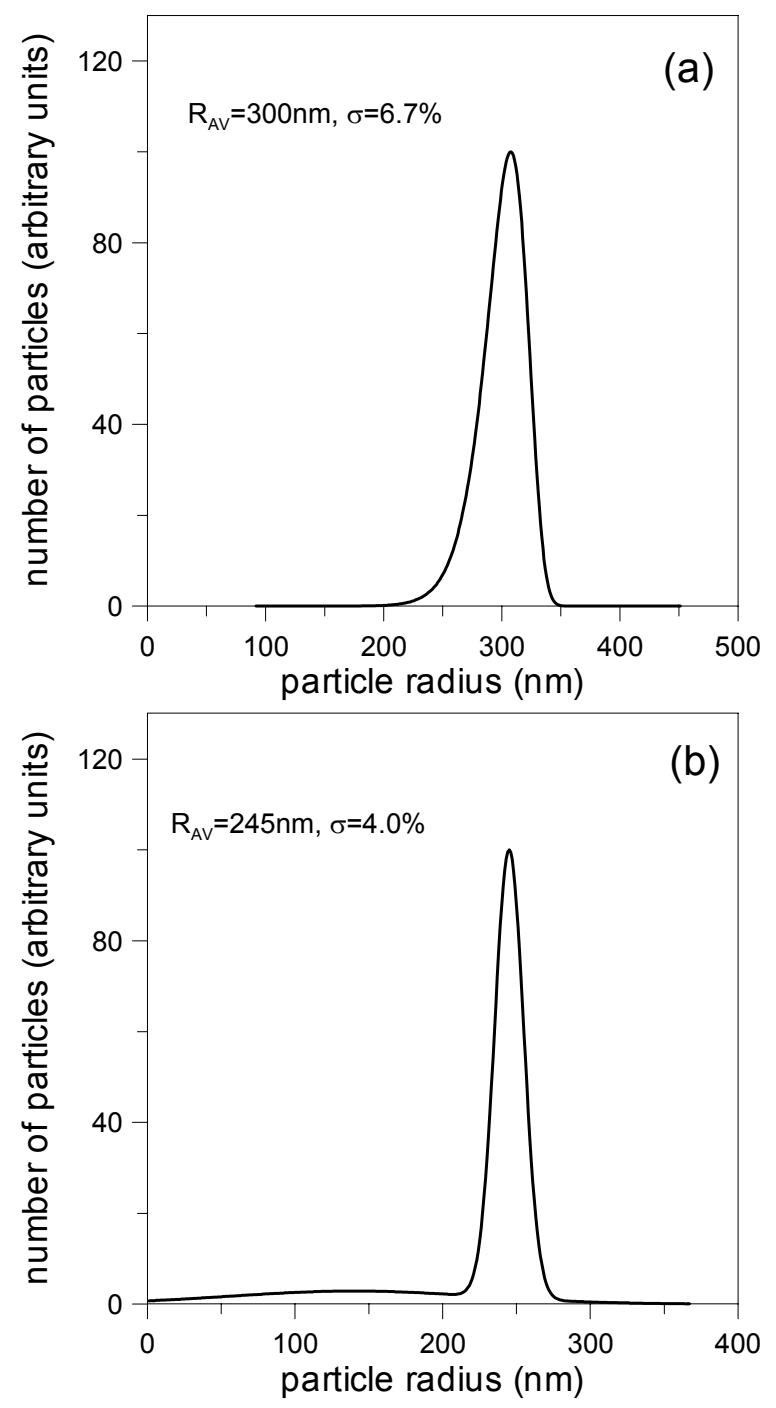

Figure 1 


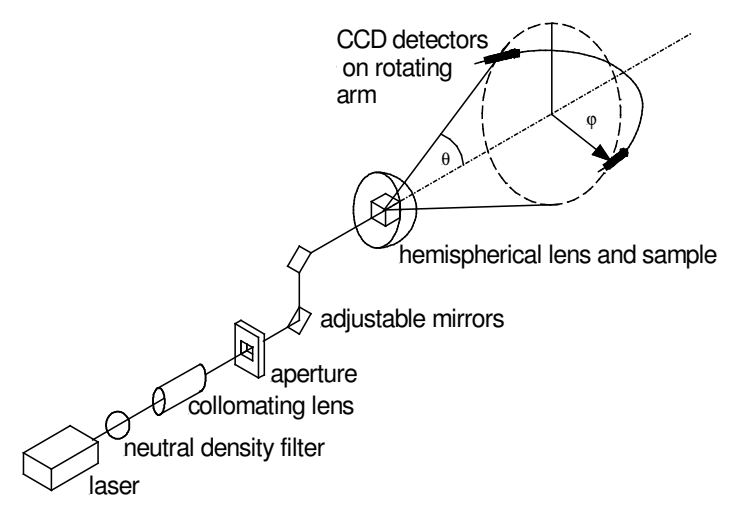

Figure 2 

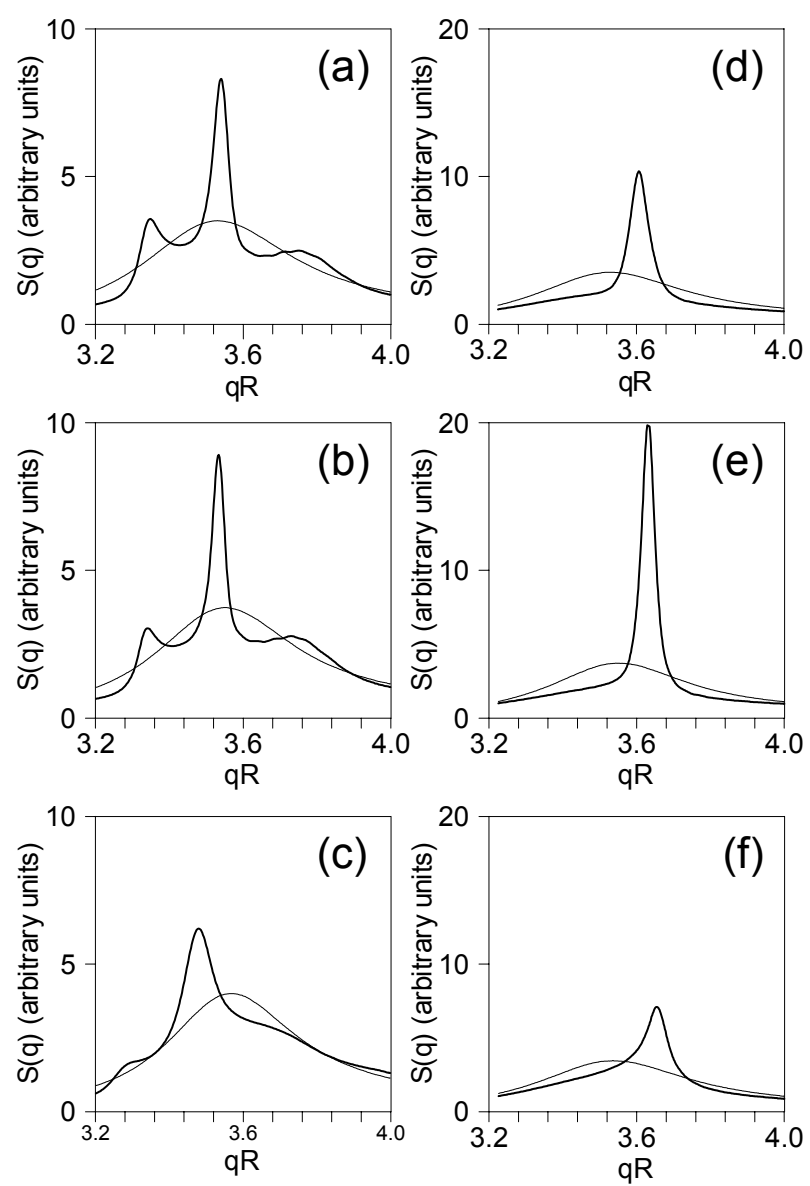

Figure 3 


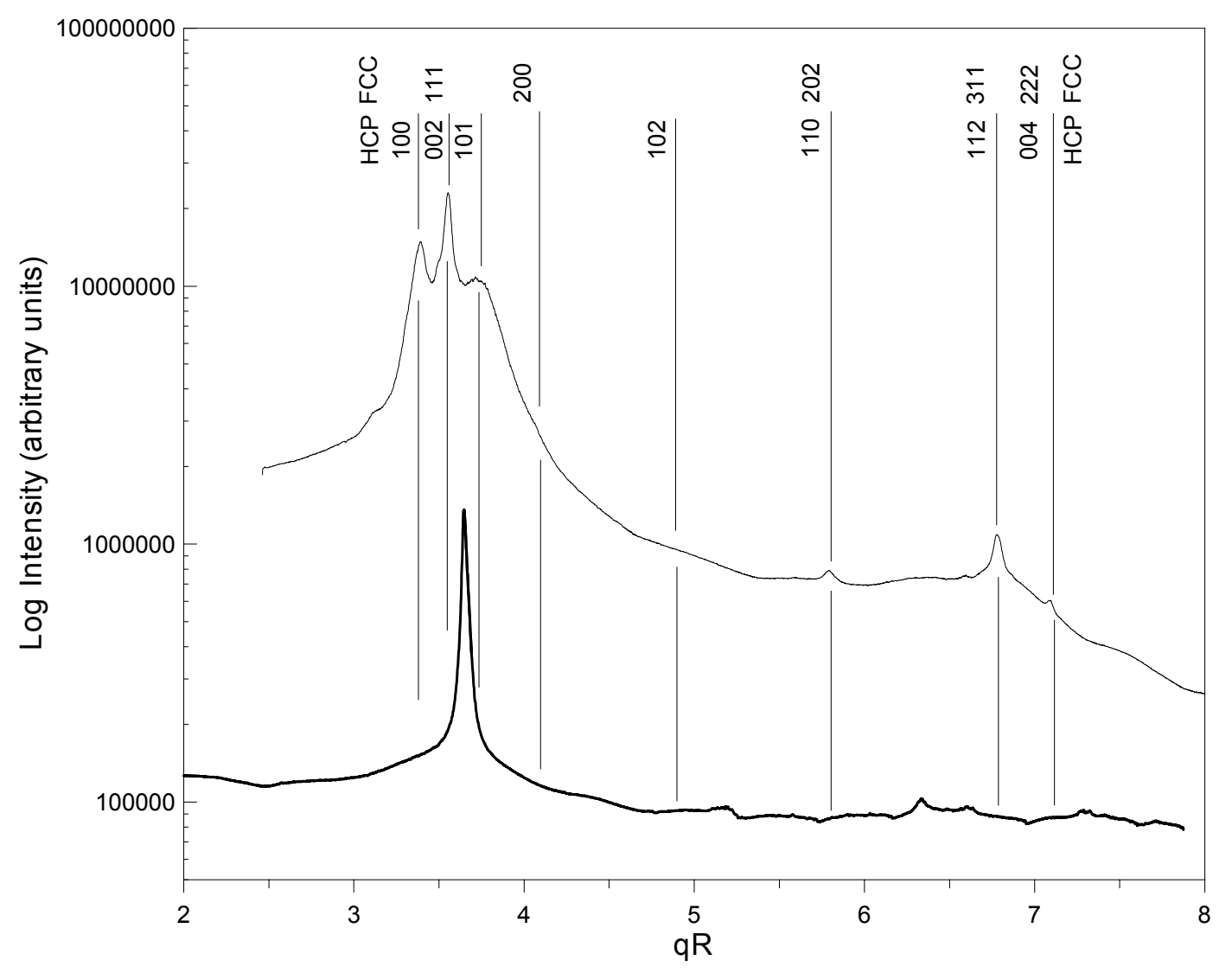

Figure 4 

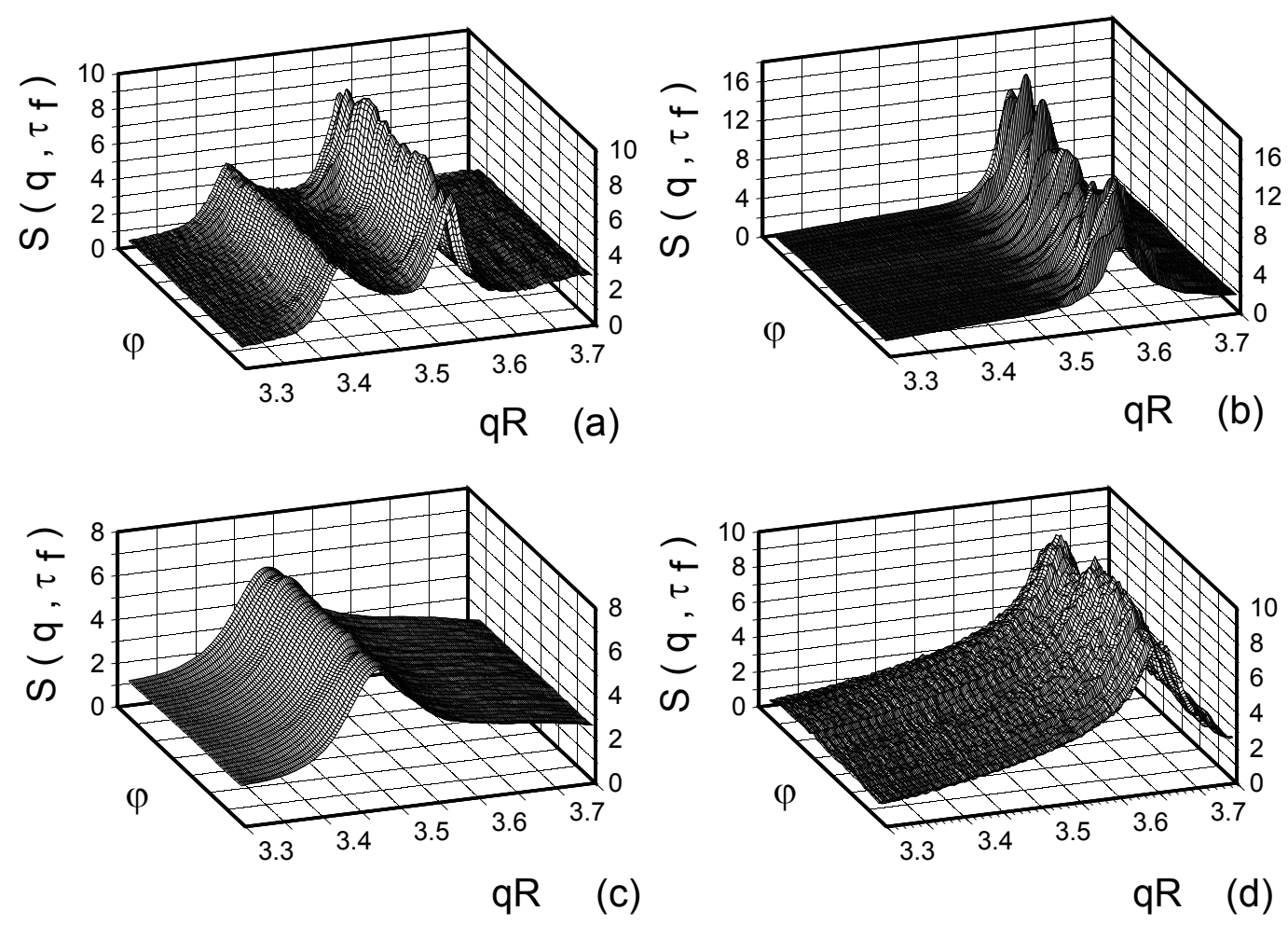

Figure 5 

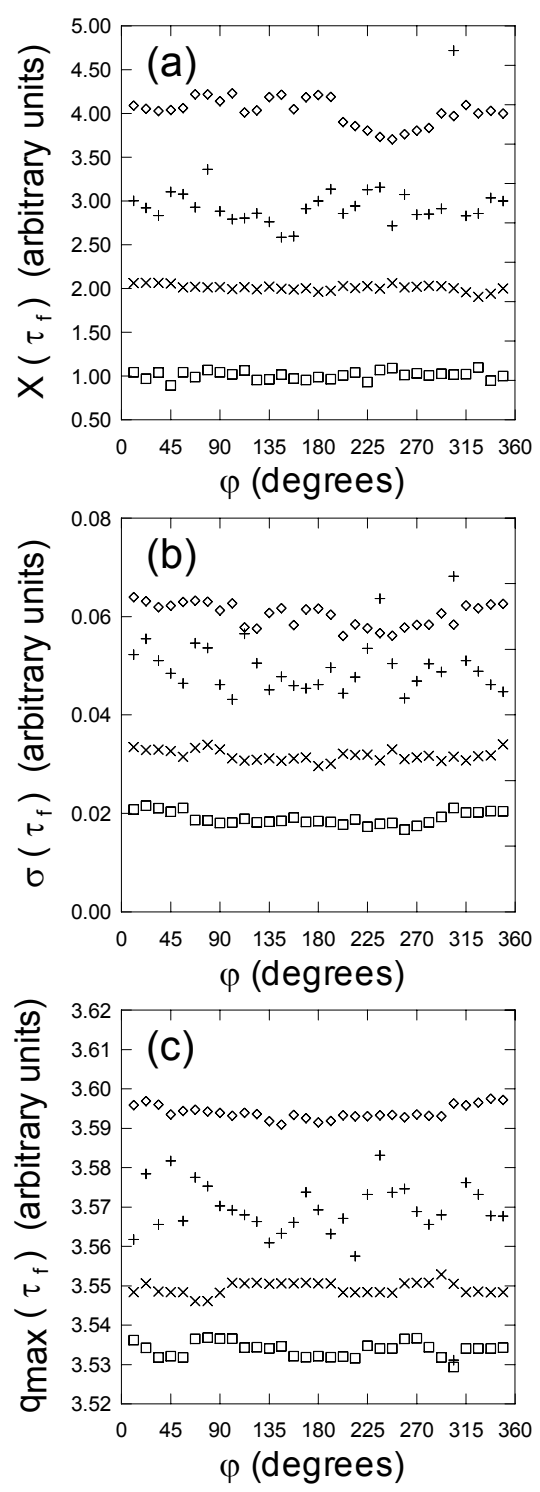

Figure 6 

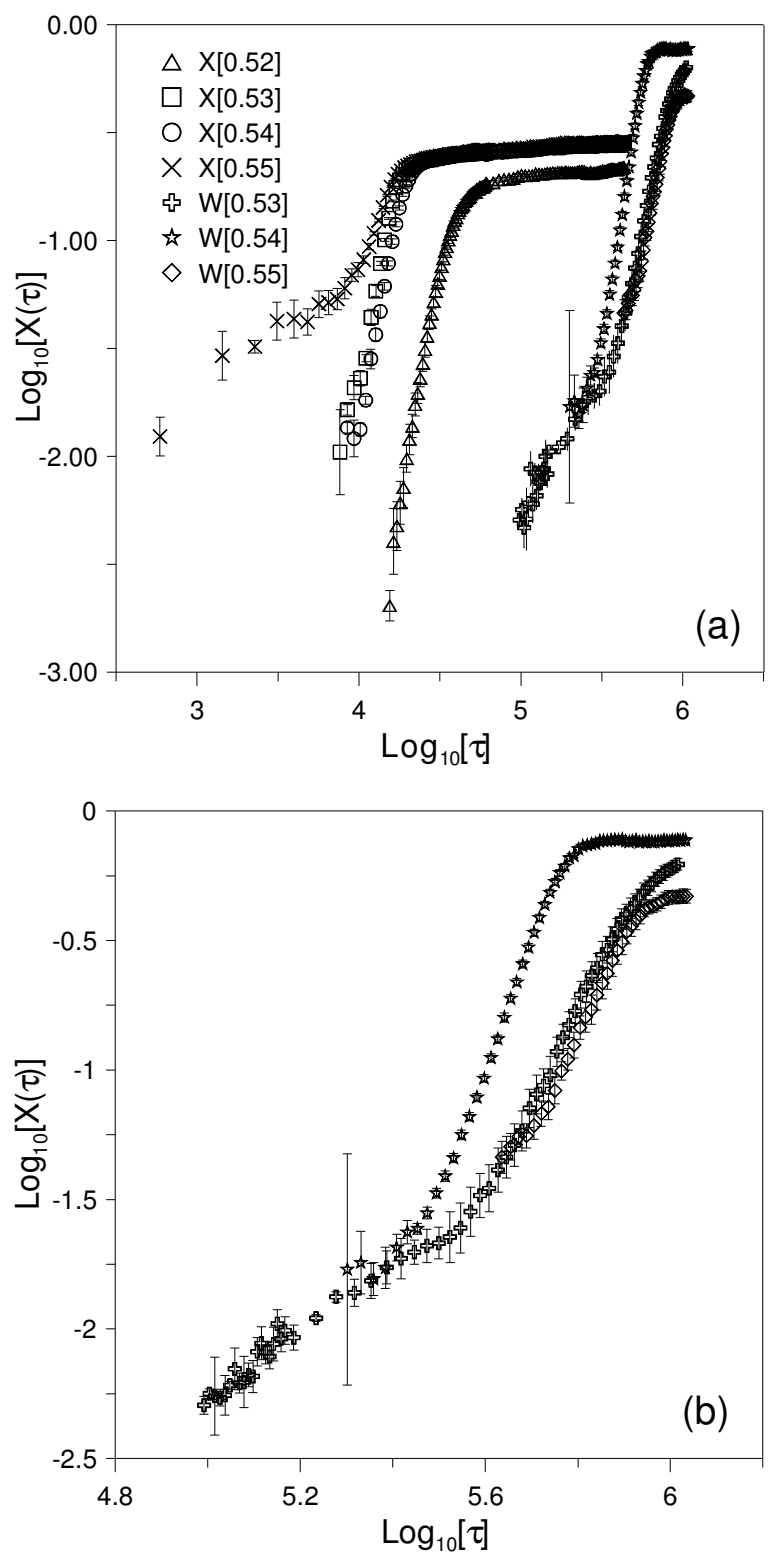

Figure 7 


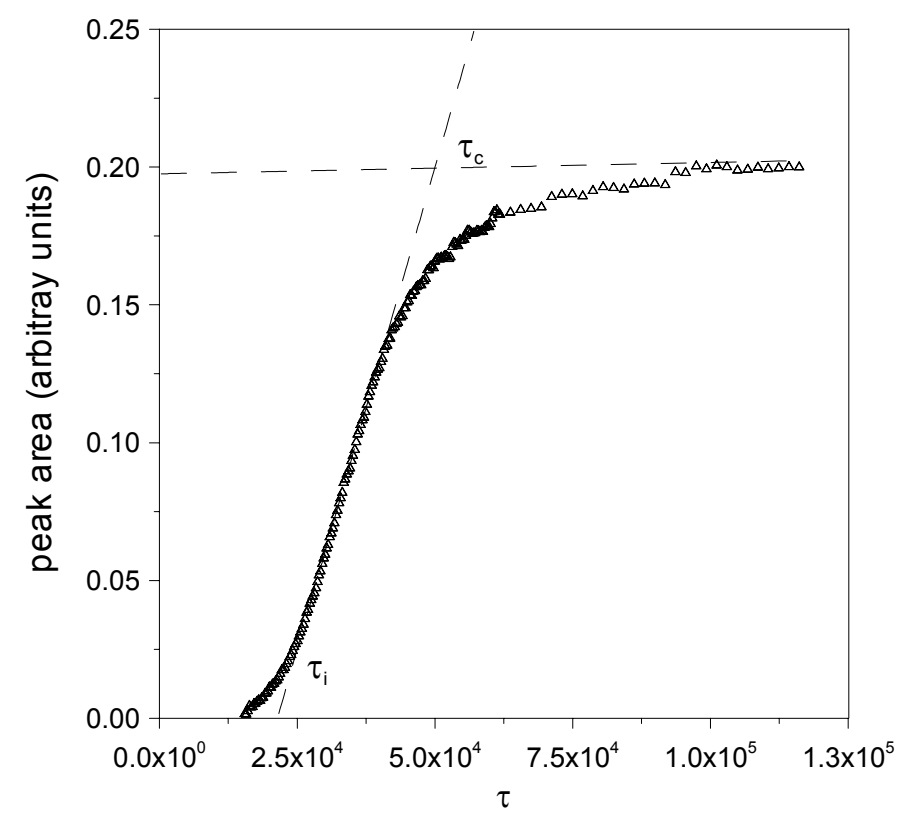

Figure 8 


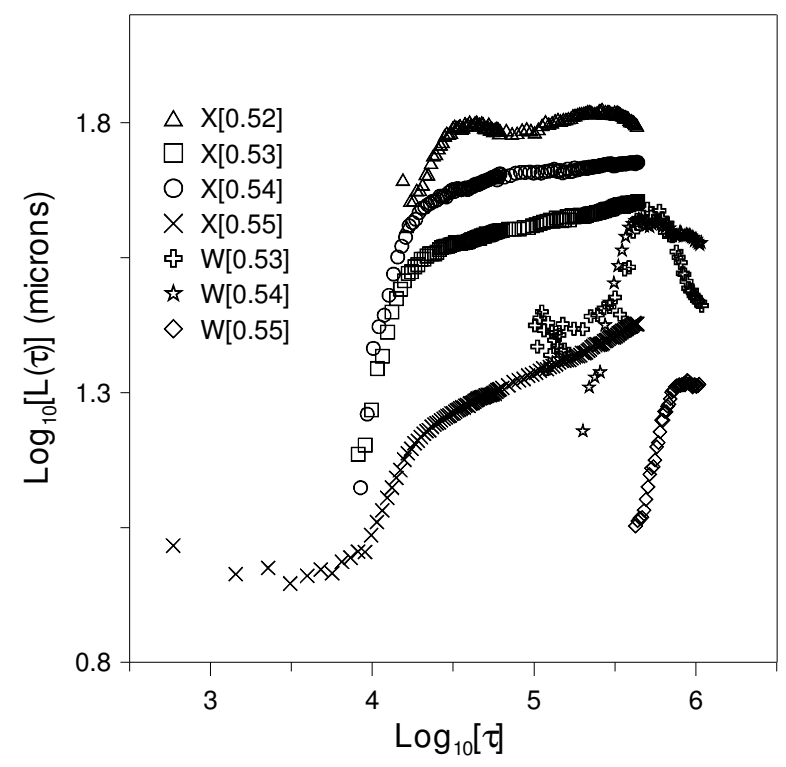

Figure 9 


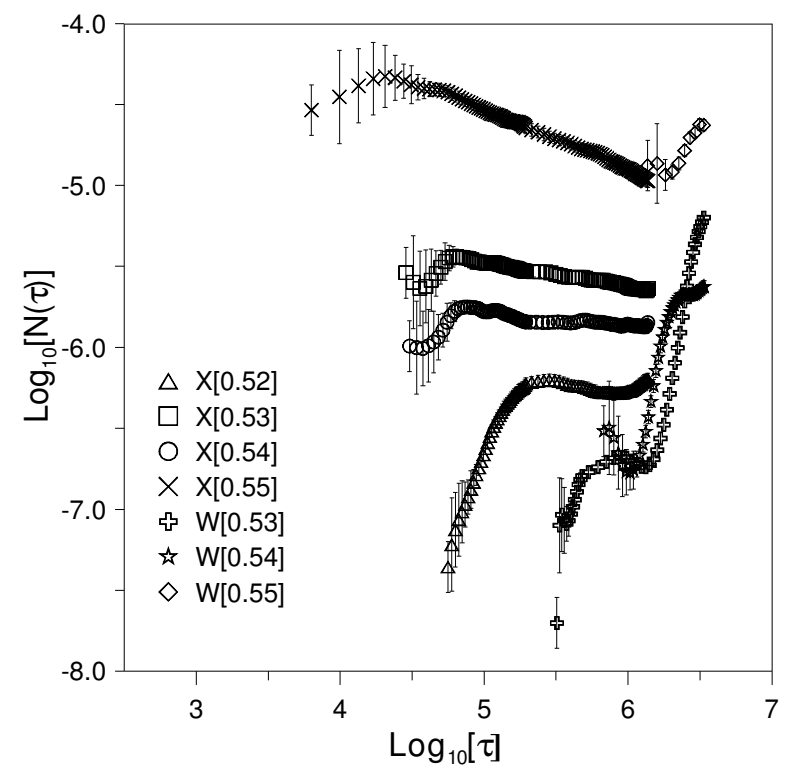

Figure 10 


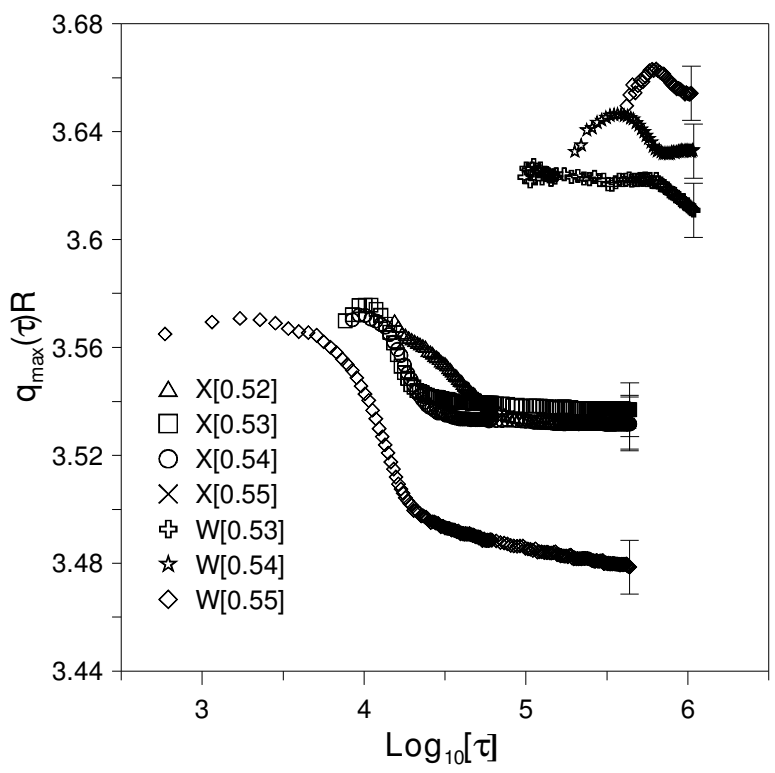

Figure 11 

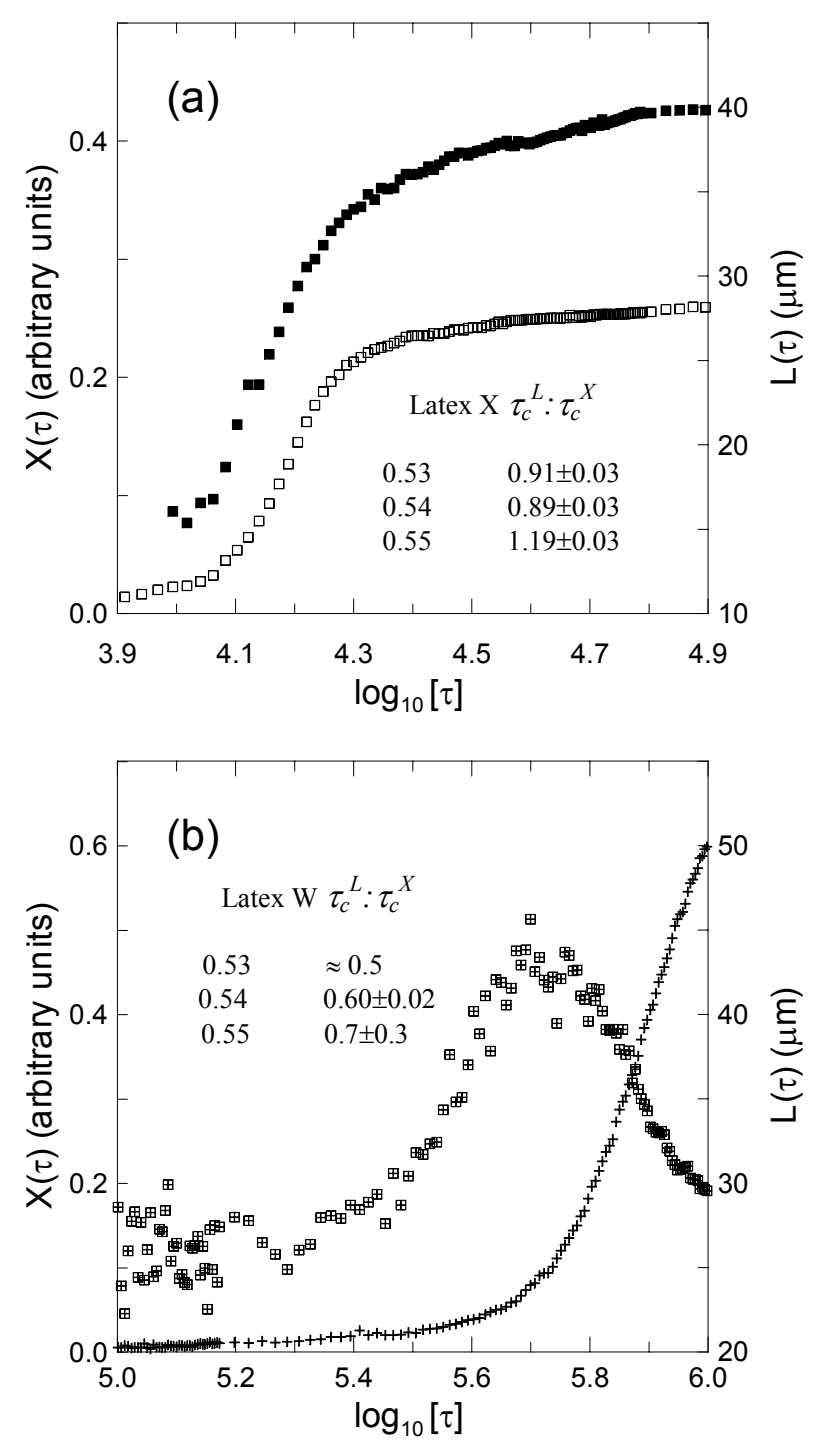

Figure 12 

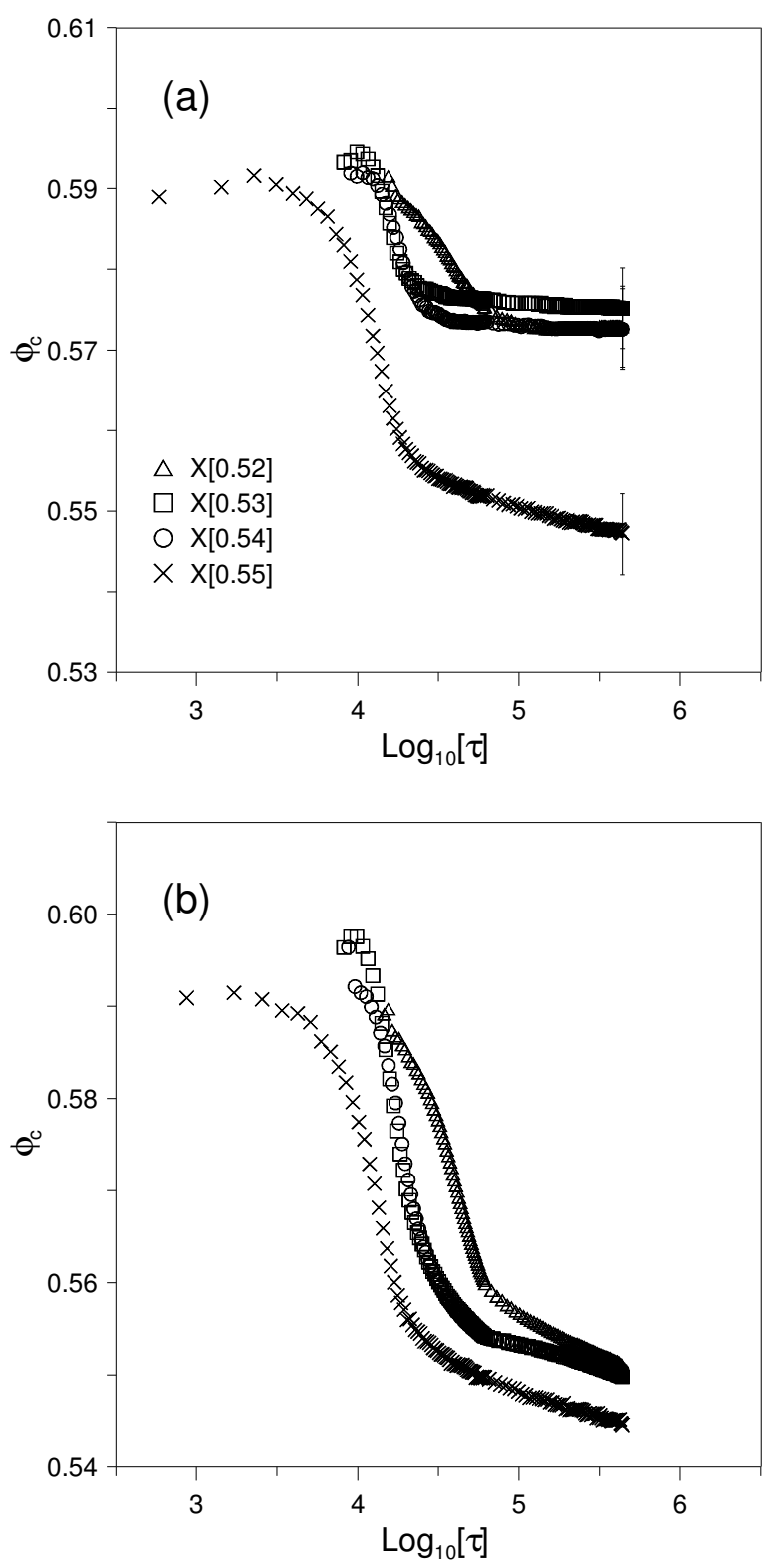

Figure 13 